Published in final edited form as:

Neurologist. 2020 November ; 25(6): 174-177. doi:10.1097/NRL.0000000000000298.

\title{
Magnetic Resonance Vessel Wall Imaging in Central Nervous System Vasculitides: a Case-Series
}

\author{
Matthew M. Padrick, MD ${ }^{1}$, Marcel M. Maya, MD², Zhaoyang Fan, PhD $^{3}$, Nicholas Szumski, \\ MD1, Patrick D. Lyden, MD ${ }^{1}$, Shlee S. Song, MD' ${ }^{1}$, Oana M. Dumitrascu, MD, MSc ${ }^{1}$ \\ ${ }^{1}$ Department of Neurology, Cedars- Sinai Medical Center, Los Angeles, CA \\ ${ }^{2}$ Department of Imaging, Cedars- Sinai Medical Center, Los Angeles, CA \\ ${ }^{3}$ Department of Biomedical Imaging Research Institute, Cedars- Sinai Medical Center, Los \\ Angeles, CA
}

\section{Abstract}

Introduction: We aim to report three cases of central nervous system (CNS) vasculitides, in which high-resolution magnetic resonance vessel wall imaging (HR-VWI) findings were instrumental in the diagnosis and management.

Case Report: Case 1: A 41-year-old obese, smoker female with arterial hypertension presented with recurrent transient ischemic attacks. Computed topography angiography demonstrated bilateral middle cerebral artery (MCA) stenosis. HR-VWI revealed uniform enhancement and thickening of the arterial wall, suggestive of MCA vasculitis. The patient reported chronic calf rash that was biopsied and revealed unspecified connective tissue disease. With immunomodulation, patient remained asymptomatic and 6-month surveillance HR-VWI showed improved MCA stenoses.

Case 2: A 56-year-old male with HSV1 encephalitis was treated with antiviral therapy and improved clinically. Two months later, the brain MRI revealed new temporo-parietal edema and distal MCA hyperintense vessels. HR-VWI showed MCA concentric smooth contrast enhancement, that was attributed to post-infectious vasculitis and had resolved on follow-up HRVWI.

Case 3: A 41-year-old male presented with one-week of headache and encephalopathy. Brain MRI revealed punctate multifocal acute ischemic infarcts and no contrast-enhancement. HR-VWI showed multifocal diffuse enhancement of distal cerebral vasculature. Patient subsequently developed branch retinal artery occlusion and hearing loss and was diagnosed with Susac's Syndrome. No recurrent symptoms were noted after immunotherapy initiation.

Conclusion: In these three cases, HR-VWI identified distinctive vascular inflammatory changes, which were crucial to guide the etiological work-up, positive diagnosis, surveillance neuroimaging, and targeted treatment. HR-VWI is an important diagnostic tool in CNS vasculitides, by providing nuanced information about arterial wall integrity and pathology.

Corresponding Author: Oana Dumitrascu MD, MSc, Cedars Sinai Medical Center. 127 S. San Vicente Blvd, A6302, Los Angeles, CA, 90048. Oana.dumitrascu@ cshs.org; Phone: 424-315-4451; Fax: 310-423-0148. 


\section{Keywords}

Cerebrovascular disease; Magnetic resonance imaging; CNS vasculitis; Vessel wall imaging

\section{Introduction}

High-resolution magnetic resonance vessel wall imaging (HR-VWI) is a non-invasive tool to assess intracranial vascular disease ${ }^{1}$. Central nervous system (CNS) vasculitis is a rare and potentially devastating cause of stroke, headache, encephalopathy, and seizure that proves a diagnostic challenge. Traditional workup includes extensive serum and cerebrospinal fluid (CSF) analyses, arterial luminal imaging with computed tomography angiography (CTA), magnetic resonance angiography (MRA), and/or digital subtraction angiography (DSA), and ultimately, if deemed necessary, a brain biopsy. No finding is specific enough to allow for a definitive diagnosis in isolation, thus more precise neuroimaging information to visualize the vessel wall inflammatory changes can prove invaluable ${ }^{2}$. HR-VWI is proposed to be a useful adjunct in the diagnostic modalities of cerebral vasculitis, particularly for large vessel vasculitis, with growing evidence for small vessel vasculitis ${ }^{1,3}$.

In our institution, HR-VWI is used in the etiologic evaluation of complex neurovascular syndromes at the discretion of the treating neurologist. We employ a whole-brain, CSFsuppressed intracranial VWI sequence based on three-dimensional turbo spin-echo with variable refocusing flip angles (SPACE), as previously reported ${ }^{4,5}$. This technique has been optimized for 3 Tesla with improved conspicuity of small vessel wall structures and, particularly, T1-mediated high-signal wall abnormalities such as inflammation-related postcontrast enhancement, thus providing a wealth of information regarding the integrity of the vessel wall itself which traditional luminal imaging modalities cannot provide. Here, we report three patients with CNS vasculitides of different etiologies, in which HR-VWI findings were instrumental in the etiological diagnosis and guided ensuing management.

\section{Case 1:}

A 41-year-old obese female with uncontrolled arterial hypertension and 20-pack-year tobacco smoking presented with recurrent left hemispheric transient ischemic attacks. MRA head demonstrated bilateral middle cerebral artery (MCA) steno-occlusive disease. Patient refused DSA due to its invasiveness. HR-VWI revealed uniform homogeneous contrast enhancement and concentric thickening of the arterial walls of bilateral MCAs, as illustrated on post-contrast T1-weighted Sampling Perfection with Application of optimized Contrasts using different flip angle Evolution (SPACE) sequence (Figure 1). Based on these imaging findings more suggestive of a vasculitic process rather than atherosclerotic plaque, detailed rheumatologic investigation and CSF analysis was undertaken. A chronic calf rash biopsy ultimately revealed chronic superficial perivascular dermatitis with focal interface change, and a diagnosis of unspecified connective tissue disease. The patient has been treated with mycophenolate mofetil in addition to antiplatelet, statin, antihypertensive agents and lifestyle modification. She had no further neurolgical symptoms. Surveillance HR-VWI performed six months after immunotherapy initiation revealed a decrement in MCA enhancement and thickening, consistent with improving vasculitis. 
Case 2:

A 56-year-old male with Herpes Simplex Virus 1 (HSV1) encephalitis was treated with intravenous acyclovir for three weeks followed by substantial clinical improvement. Two months after the initial diagnosis, a surveillance brain MRI showed new temporo-parietal lobe edema with contrast-enhancement, which was thought to be consistent with infarction. In the same region, the MCA showed hyperintense branches that were suggestive of slow flow (Figure 2). HR-VWI revealed concentric smooth contrast enhancement in the respective MCA branches. They were thought to be due to a post-infectious vasculitic process, as HSV1 CSF and serum PCR remained negative. As the patient was stable clinically, the decision was made to monitor with serial HR-VWI, keeping a low threshold to resume corticosteroid and antiviral therapy. Follow-up HR-VWI one month later showed resolution of vessel wall enhancement and vasculitic changes, whereas the patient remained clinically stable.

\section{Case 3:}

A 41-year-old male with no medical history presented with one week history of progressive headache and encephalopathy. Brain MRI revealed scattered punctate acute cerebral infarctions in multiple vascular territories. Embolic workup and DSA were unremarkable. Ensuing brain MRI with contrast performed two days after the initial scan for transitory speech changes, showed an increased number of punctate infarcts, and no abnormal contrast enhancement. HR-VWI showed diffuse contrast enhancement of distal cerebral vasculature (Figure 3), prompting further infectious and rheumatologic investigations, and an expedited brain biopsy, that was unrevealing. Patient developed right eye branch retinal artery occlusion and hearing deficits approximately one week after the initial presentation. He was diagnosed with Susac's Syndrome (SS), and will be maintained on cyclophosphamide and intravenous immunoglobulin for at least six months.

\section{Discussion}

Etiological diagnosis of intracranial vasculopathies remains challenging. Whereas traditional angiographic modalities provide information regarding vessel lumen appearance, they are limited in the assessment of arterial wall integrity and pathology. Conversely, the underlying vasculitic process has typical imaging characteristics on HR-VWI, as illustrated in our caseseries and prior reports ${ }^{3,6-14}$. In large vessel vasculitis, HR-VWI often demonstrates smooth, homogeneous, concentric arterial wall thickening and contrast enhancement ${ }^{3,7,15}$, as seen in our first two cases. The enhancement can be multifocal and segmental, affecting the stenotic or non-stenotic areas ${ }^{15}$. Leptomeningeal enhancement was similarly described ${ }^{16}$. In small vessel vasculitis, the vessel imaging literature is currently sparse ${ }^{3}$. In our last patient, diagnosed with a rare form of autoimmune small vessel vasculitis/ endotheliopathy, HR-VWI illustrated multifocal enhancement of the pial vascular structures and small vessels.

In our first case, HR-VWI findings led to a diagnosis of mixed connective tissue disease prompting immunotherapy, in a patient with CTA findings that in many instances would be attributed to atherosclerosis given the patient's vascular risk factors. In our second case, HR- 
VWI revealed unexpected ongoing arteriopathy, and provided a noninvasive modality to track HSV1 vasculitis progression and ultimate resolution. In our last case, recognition of distal vasculitic changes on HR-VWI prompted immunotherapy with intravenous corticosteroids and accelerated consideration for brain biopsy.

Not all that enhances is vasculitis, however, as atherosclerotic plaque will also enhance if the vessel wall is inflamed. The two distinct entities can be differentiated by their pattern of enhancement: the typical HR-VWI findings of atherosclerotic plaque are inherently heterogeneous, eccentric (irregular) with differing degrees of enhancement, while vasculitis is associated with concentric smooth vascular wall enhancement ${ }^{9,17,18}$. Similarly, lack of enhancement should not completely rule out a vasculitic process, although it should prompt the clinician to consider a noninflammatory entity such as reversible cerebral vasoconstriction syndrome (RCVS). RCVS should have no contrast enhancement, as there is no inflammation of the vessel wall itself. Rarely, RCSV might exhibit minimal or mild enhancement, compared to with the typical intense wall enhancement in active vasculitis ${ }^{15,} 19$.

Furthermore, HR-VWI could be used to monitor the disease activity, as decreased vessel wall enhancement was recently proposed as a biomarker of response to immunomodulatory treatment in patients with CNS vasculitis ${ }^{13,20}$.

Regarding small vessel disease, Zeiler at al. highlighted that HR-VWI may be able not only to identify distal vascular inflammation but also to direct open biopsies of intracranial target vessels and adjacent brain parenchyma ${ }^{3}$. HR-VWI findings in SS similar to our case were not found in the literature. A letter to the editor recently reported 3 patients with SS, whose HR-VWI either exhibited no abnormality or leptomeningeal cerebellar enhancement ${ }^{21}$. Hence, the application of HR-VWI in small vessel CNS vasculitides needs to be further explored.

HR-VWI emerges as essential adjunct to a timely positive diagnosis of CNS vasculitis, by identifying specific inflammatory features and differentiating them from atherosclerotic plaque, RCVS, dissections and other non-inflammatory non-atherosclerotic vasculopathies such as moyamoya ${ }^{1,22,23}$. This diagnostic tool may play a role in cryptogenic stroke workup by identifying stroke syndromes due to inflammatory vasculopathy 24,25 . Additionally, it may provide a noninvasive modality to track intracranial vasculopathy progression and response to treatment, as well as to identify a peripherally located inflamed vessel to target for biopsy 1,3 . Given the extensive healthcare costs and inherent risks associated with diagnosing cerebral vasculitis, including invasive procedures such as DSA and brain biopsy, a future prospective diagnostic utility study to determine actionability of HR-VWI findings is certainly warranted.

\section{Conclusions:}

Clinicians taking care of patients with CNS vasculitis should be familiar with HR-VWI applications, diagnostic and monitoring value. As more institutions incorporate HR-VWI in their neuroimaging armamentarium, more associations between neuroimaging findings and specific disease processes may become apparent to further guide clinical practice. 


\section{Study Funding:}

supported in part by NIH/NHLBI 1R01 HL147355

Conflicts of Interest: Drs. Fan and Song are funded by NIH/NHLBI 1R01 HL147355; Dr. Lyden is funded by NIH, U24NS113452 and R01 NS07930. Dr. Lyden receives royalties from the book Thrombolytic Therapy for Acute Ischemic Stroke, 3rd ed. Drs. Padrick, Maya, Szumski and Dumitrascu have no relevant financial disclosures.

\section{References}

1. Mandell DM, Mossa-Basha M, Qiao Y, et al. Intracranial Vessel Wall MRI: Principles and Expert Consensus Recommendations of the American Society of Neuroradiology. AJNR Am J Neuroradiol 2017;38:218-229. [PubMed: 27469212]

2. Hajj-Ali RA, Calabrese LH. Central nervous system vasculitis: advances in diagnosis. Curr Opin Rheumatol 2020;32:41-46.

3. Zeiler SR, Qiao Y, Pardo CA, Lim M, Wasserman BA. Vessel Wall MRI for Targeting Biopsies of Intracranial Vasculitis. AJNR Am J Neuroradiol 2018;39:2034-2036. [PubMed: 30262647]

4. Fan Z, Yang Q, Deng Z, et al. Whole-brain intracranial vessel wall imaging at 3 Tesla using cerebrospinal fluid-attenuated T1-weighted 3D turbo spin echo. Magn Reson Med 2017;77:11421150. [PubMed: 26923198]

5. Yang Q, Deng Z, Bi X, et al. Whole-brain vessel wall MRI: A parameter tune-up solution to improve the scan efficiency of three-dimensional variable flip-angle turbo spin-echo. J Magn Reson Imaging 2017;46:751-757. [PubMed: 28106936]

6. Khoury JA, Hoxworth JM, Mazlumzadeh M, Wellik KE, Wingerchuk DM, Demaerschalk BM. The clinical utility of high resolution magnetic resonance imaging in the diagnosis of giant cell arteritis: a critically appraised topic. Neurologist 2008;14:330-335. [PubMed: 18784606]

7. Wang LJ, Kong DZ, Guo ZN, Zhang FL, Zhou HW, Yang Y. Study on the Clinical, Imaging, and Pathological Characteristics of 18 Cases with Primary Central Nervous System Vasculitis. J Stroke Cerebrovasc Dis 2019;28:920-928. [PubMed: 30635219]

8. Van Rooij JL, Rutgers DR, Spliet WG, Frijns CJ. Vessel wall enhancement on MRI in the diagnosis of primary central nervous system vasculitis. Int J Stroke 2018;13:NP24-NP27. [PubMed: 30102571]

9. Mossa-Basha M, Shibata DK, Hallam DK, et al. Added Value of Vessel Wall Magnetic Resonance Imaging for Differentiation of Nonocclusive Intracranial Vasculopathies. Stroke 2017;48:30263033. [PubMed: 29030476]

10. Tsivgoulis G, Lachanis S, Magoufis G, Safouris A, Kargiotis O, Stamboulis E. High-Resolution Vessel Wall Magnetic Resonance Imaging in Varicella-Zoster Virus Vasculitis. J Stroke Cerebrovasc Dis 2016;25:e74-76. [PubMed: 27067878]

11. Guerrero WR, Dababneh H, Hedna S, Johnson JA, Peters K, Waters MF. Vessel wall enhancement in herpes simplex virus central nervous system vasculitis. J Clin Neurosci 2013;20:1318-1319. [PubMed: 23517674]

12. Brinjikji W, Lehman V, Huston J 3rd, Luetmer PH, Lanzino G, Rabinstein AA. Decreased vessel wall enhancement as a biomarker for response to corticosteroids in a patient with CNS vasculitis. J Neurosurg Sci. Italy 2019: 100-101.

13. Tsivgoulis G, Papadimitropoulos GN, Lachanis S, et al. High-resolution Intracranial Vessel Wall Imaging in Monitoring Treatment Response in Primary CNS Angiitis. Neurologist 2018;23:188190. [PubMed: 30379741]

14. Destrebecq V, Sadeghi N, Lubicz B, Jodaitis L, Ligot N, Naeije G. Intracranial Vessel Wall MRI in Cryptogenic Stroke and Intracranial Vasculitis. J Stroke Cerebrovasc Dis 2020;29:104684. [PubMed: 32151477]

15. Mandell DM, Matouk CC, Farb RI, et al. Vessel wall MRI to differentiate between reversible cerebral vasoconstriction syndrome and central nervous system vasculitis: preliminary results. Stroke 2012;43:860-862. [PubMed: 22156692] 
16. Eiden S, Beck C, Venhoff N, et al. High-resolution contrast-enhanced vessel wall imaging in patients with suspected cerebral vasculitis: Prospective comparison of whole-brain 3D T1 SPACE versus 2D T1 black blood MRI at 3 Tesla. PLoS One 2019;14:e0213514. [PubMed: 30849127]

17. Zhang L, Zhang N, Wu J, Huang Y, Liu X, Chung YC. High resolution three dimensional intracranial arterial wall imaging at $3 \mathrm{~T}$ using T1 weighted SPACE. Magn Reson Imaging 2015;33:1026-1034. [PubMed: 26143482]

18. Zhao DL, Deng G, Xie B, et al. High-resolution MRI of the vessel wall in patients with symptomatic atherosclerotic stenosis of the middle cerebral artery. J Clin Neurosci 2015;22:700704. [PubMed: 25744074]

19. Obusez EC, Hui F, Hajj-Ali RA, et al. High-resolution MRI vessel wall imaging: spatial and temporal patterns of reversible cerebral vasoconstriction syndrome and central nervous system vasculitis. AJNR Am J Neuroradiol 2014;35:1527-1532. [PubMed: 24722305]

20. Brinjikji W, Lehman V, Huston J 3rd, Luetmer PH, Lanzino G, Rabinstein AA. Decreased vessel wall enhancement as a biomarker for response to corticosteroids in a patient with CNS vasculitis. $\mathrm{J}$ Neurosurg Sci 2019;63:100-101. [PubMed: 28240529]

21. Lehman VT, Klaas JP, Makol A, et al. High-resolution vessel wall imaging in Susac's Syndrome. J Neurosurg Sci 2019;63:235-236. [PubMed: 28959874]

22. Mossa-Basha M, Hwang WD, De Havenon A, et al. Multicontrast high-resolution vessel wall magnetic resonance imaging and its value in differentiating intracranial vasculopathic processes. Stroke 2015;46:1567-1573. [PubMed: 25953365]

23. Park MS, Cha J, Chung JW, Seo WK, Kim GM, Bang OY. Arterial Dissection as a Cause of Intracranial Stenosis in East Asians. J Am Coll Cardiol 2017;70:2205-2206. [PubMed: 29050569]

24. Schaafsma JD, Rawal S, Coutinho JM, et al. Diagnostic Impact of Intracranial Vessel Wall MRI in 205 Patients with Ischemic Stroke or TIA. AJNR Am J Neuroradiol 2019;40:1701-1706. [PubMed: 31488500]

25. Kesav P, Krishnavadana B, Kesavadas C, et al. Utility of intracranial high-resolution vessel wall magnetic resonance imaging in differentiating intracranial vasculopathic diseases causing ischemic stroke. Neuroradiology 2019;61:389-396. [PubMed: 30637463] 

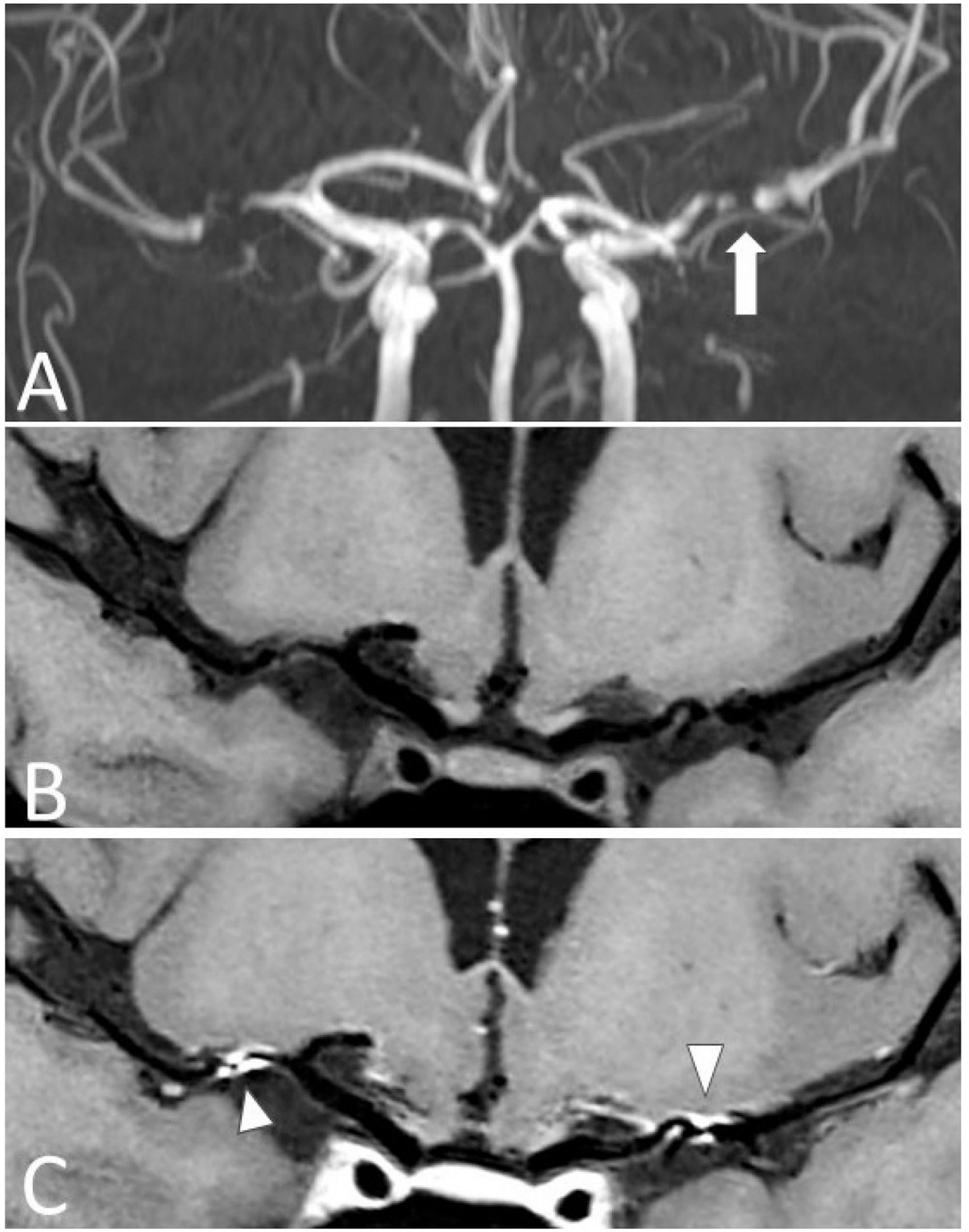

Figure 1:

3D Time-of-Flight brain MR angiogram shows bilateral middle cerebral artery (MCA) M1 stenosis. Note dilatation alternating segments of stenosis and dilatation on the left side (white arrow) (A). Brain MRI coronal T1-weighted SPACE without (B) and with contrast (C) shows concentric wall thickening and contrast enhancement in the MCA bilaterally (arrowheads). 

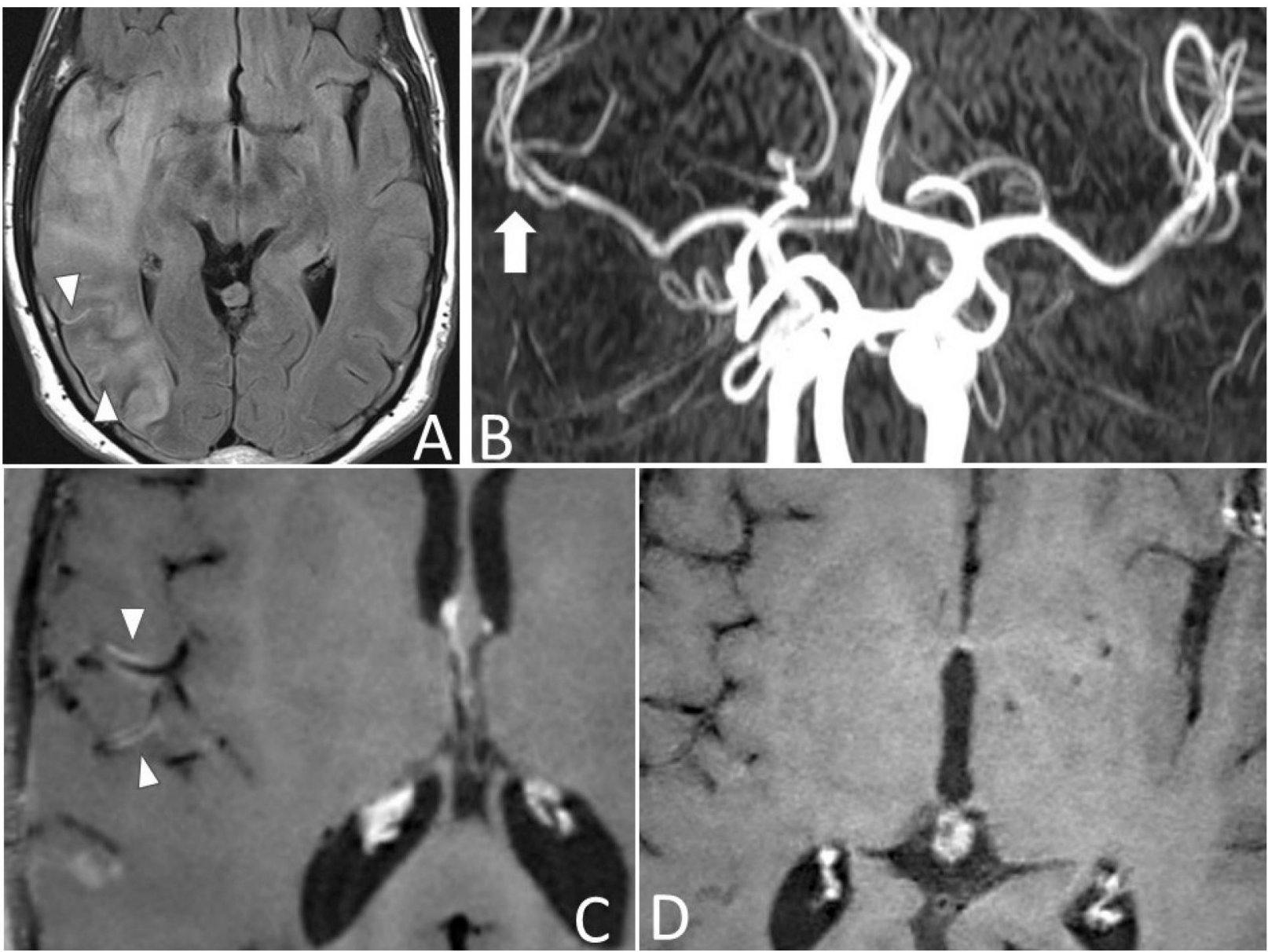

Figure 2:

MRI brain axial FLAIR showing temporal lobe edema and hyperintense vessels (arrowheads) suggestive of ischemia and slow flow in the MCA branches (A). MRA head coronal MIP images show focal irregularities in the right MCA branches (white arrow) (B). HR-VWI axial T1-weighted SPACE image shows concentric wall thickening and contrast enhancement in same MCA branches (arrowheads) (C). At one-month follow-up, HR-VWI shows resolution of contrast enhancement (D). 

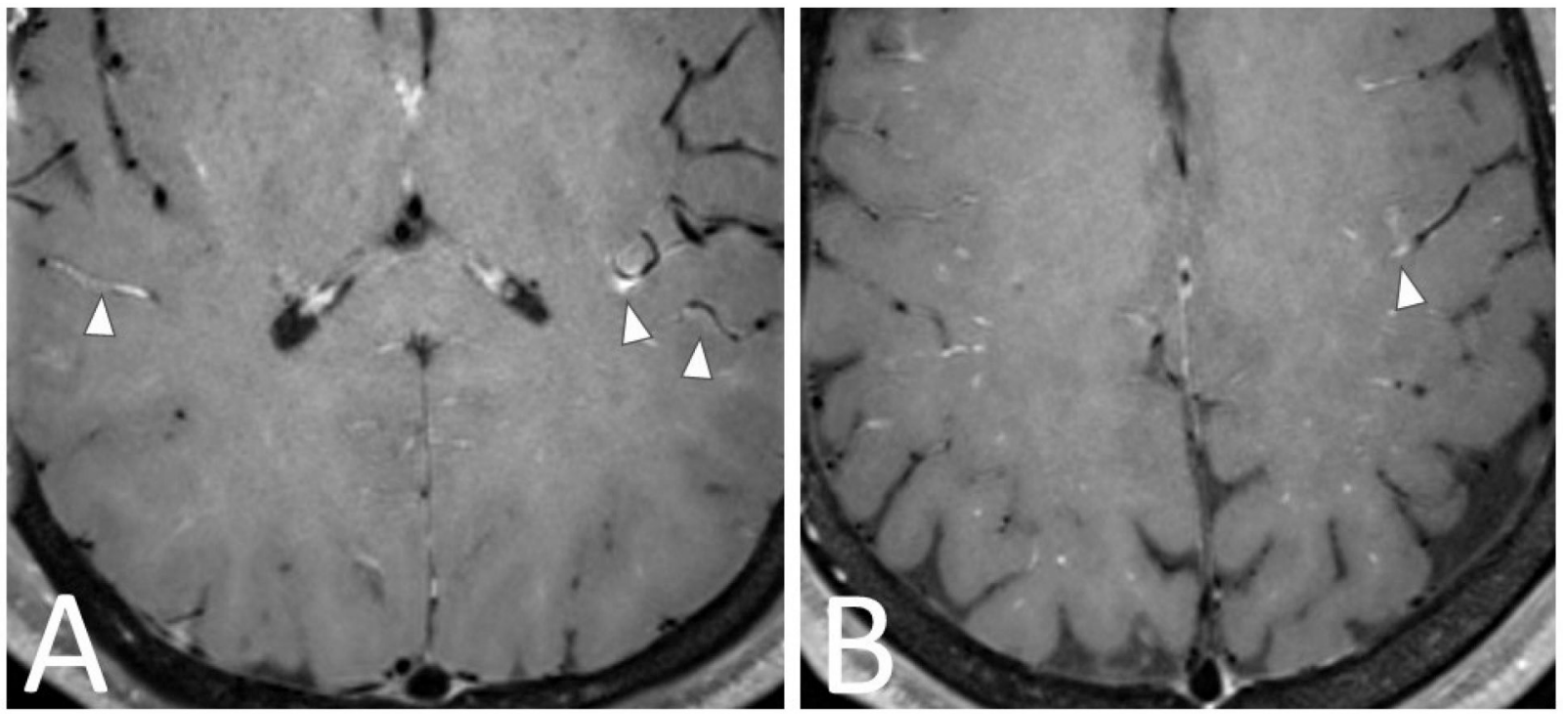

Figure 3:

Brain MR axial T1- weighted SPACE post contrast images show multifocal enhancement of the pial vascular structures and small vessels (arrowheads). 